\title{
Nutzung von Routinedaten für die Qualitätsmessung in Österreich
}

\section{G. Fülöp}

Qualitätsmanagement

Schlüsselwörter

Ergebnisqualität

Gesundheitswesen

Österreich

Daten

Keywords

outcome quality

health care

Austria

data

Institut

Gesundheit Österreich GmbH/ Bundesinstitut für Gesundheitswesen (GÖG/ÖBIG), Wien, Österreich

\section{Bibliografie}

Dol $10.1055 / s-0029-1242664$ Dtsch Med Wochenschr 2009; 134: S308 - @ Georg Thieme Verlag KG Stuttgart · New York . ISSN 0012-0472

\section{Korrespondenz}

\section{Dr. Gerhard Fülöp}

Gesundheit Österreich $\mathrm{GmbH}$ / Bundesinstitut für Gesundheits wesen (GÖG/ÖBIG)

Stubenring 6

1010 Wien

Tel. +43 151561-163

eMail gerhard.fueloep@goeg.at
Die Nutzung von Routinedaten für die Qualitätsmessung (QMR) wurde in Österreich auf Bundesebene bisher kaum thematisiert, obwohl zumindest für den akutstationären Bereich seit der Einführung der Leistungsorientierten Krankenanstaltenfinanzierung (LKF) im Jahr 1997 umfangreiche Routinedaten zur Verfügung stehen, die für die QMR genutzt werden könnten (Krankenanstalten-Statistik, Diagnosen- und Leistungsdokumentation). Für den ambulanten Bereich bleibt noch zu klären, ob auch die in diesem Bereich in Österreich dokumentierten Routinedaten (Abrechnungsdaten der Sozialversicherung, Inanspruchnahme-Daten der Spitalsambulanzen) für QMR nutzbar wären.

Der Österreichische Strukturplan Gesundheit 2008 (ÖSG 2008), der den Rahmen für die Regionalen Strukturpläne Gesundheit der 9 Bundesländer bildet, konzentriert sich auf Vorgaben zur Strukturqualität, soll aber in der Zukunft gezielt um die Bereiche Prozess- und Ergebnisqualität erweitert werden. Allerdings entstehen im Rahmen der im ÖSG verankerten „Leistungsangebotsplanung“ bereits jetzt Fragestellungen, zu deren Lösung Ergebnisse aus der QMR beitragen könnten. Dazu zählt insbesondere auch der empirische Nachweis von Zusammenhängen zwischen dem Erreichen einer jährlichen „Mindestfrequenz" in bestimmten Leistungsbereichen und der Ergebnisqualität.

Wenn dieser Nachweis geführt werden kann und derartige Zusammenhänge zusätzlich auch in der internationalen Literatur belegt sind, so erhöht sich die Wahrscheinlichkeit, dass einige der - derzeit im ÖSG für eine Reihe von Indikationsgruppen nur unverbindlich festgelegten - „Mindestfrequenzen“ als verbindlich deklariert werden. Dies hätte (ähnlich wie die „Mindestmengenvereinbarung“ in Deutschland) für viele Spitäler zur Konsequenz, dass die niedrigfrequente Erbringung spezieller Leistungen aus Gründen der Qualitätssicherung eingestellt werden muss und sich in der Folge die - im ÖSG für mehrere Bereiche definierten - „Referenzzentren“ rascher herausbilden würden. Insofern könnten also klare Ergebnisse aus der QMR indirekt zur im ÖSG angestrebten überregionalen Bündelung bestimmter spezieller Leistungen beitragen.

Nachdem die erwähnten Daten aus der Krankenanstalten-Statistik in den Datenbanken der Gesundheit Österreich GmbH in Form von anonymisierten Einzeldatensätzen für den gesamten Zeitraum 1992-2008 verfügbar sind, wurden diese Daten erstmals testweise für die Analyse von Zusammenhängen zwischen den jährlichen Leistungsmengen und der Ergebnisqualität (gemessen am Anteil der postoperativ noch im Spital verstorbenen Patienten/-innen) verwendet. Dabei gelangten vorerst nur einfachste statistische Verfahren zur Anwendung. Am Beispiel der Eingriffe am Pankreas bzw. am Ösophagus (beide Gruppen sind auch in der o.a. „Mindestmengenvereinbarung“ enthalten) zeigten sich in dieser Grobanalyse statistische Zusammenhänge, die die international bereits bekannten Zusammenhänge zwischen Leistungsmenge und intramuraler Sterblichkeit auch für Österreich bestätigen (vgl. [3]).

Aus den ersten Diskussionen dieser Ergebnisse mit Experten/-innen ist zu schließen, dass es noch einer umfangreichen Weiterentwicklung bzw. Differenzierung der Methoden und Indikatoren bedarf, bevor diese ersten Ergebnisse als gesichert betrachtet werden können. Weiterhin stellt sich die Frage, ob nicht wirklich verlässliche Ergebnisse ausschließlich aus jenen „Ergebnisqualitätsregistern“ zu erwarten sind, die sich in Österreich seit einigen Jahren im Aufbau bzw. im Betrieb befinden (z.B. „Stroke Unit Register“, „Endoprothesen-Register“). In der GÖG soll an der Beantwortung dieser Frage für Österreich gearbeitet werden, wobei derzeit davon ausgegangen wird, dass sich QMR und „Ergebnisqualitätsregister" nicht gegenseitig ausschließen, sondern vielmehr gegenseitig ergänzen sollten.

Eine weitere Fragestellung wäre, ob bzw. wann Österreich dem Beispiel der Spitäler in Deutschland folgen wird und Ergebnisse der QMR in den Spitälern via Internet (z.B. im Spitalskompass, vgl. www.spitalskompass.at) zur Veröffentlichung freigibt.

Autorenerklärung: Der Autor erklärt, dass keine relevanten finanziellen Verbindungen in Bezug auf dieses Manuskript bestehen.

\section{Literatur}

1 BMG/GÖG/ÖBIG. Österreichischer Strukturplan Gesundheit 2008. Wien: 2009

2 Oberaigner W, Stühlinger W. Influence of Department Volume on Cancer Survival for Gynaecological Cancers - A population-based study in Tyrol, Austria. Gynecologic Oncology 2 2006; 103: 527-534

3 Van Heek NT et al. Hospital Volume and Mortality after Pancreatic Resection - a Systematic Review and an Evaluation of Intervention in the Netherlands. Annals of Surgery 6 2005; 242: 781-790 